\title{
On the Independence Numbers of the Cubes of Odd Cycles
}

\author{
Tom Bohman * \\ Department of Mathematical Sciences \\ Carnegie Mellon University \\ Pittsburgh, U.S.A. \\ tbohman@moser.math.cmu.edu
}

\author{
Ron Holzman ${ }^{\dagger}$ \\ Department of Mathematics \\ Technion-Israel Institute of Technology \\ Haifa, Israel \\ holzman@tx.technion.ac.il
}

\author{
Venkatesh Natarajan \\ Department of Mathematical Sciences \\ Carnegie Mellon University \\ Pittsburgh, U.S.A.
}

Submitted: Aug 3, 2012; Accepted: July 24, 2013; Published: Aug 2, 2013

Mathematics Subject Classifications: 05C38, 05C69

\begin{abstract}
We give an upper bound on the independence number of the cube of the odd cycle $C_{8 n+5}$. The best known lower bound is conjectured to be the truth; we prove the conjecture in the case $8 n+5$ prime and, within 2 , for general $n$.
\end{abstract}

\section{Introduction}

Let $G_{1}=\left(V_{1}, E_{1}\right)$ and $G_{2}=\left(V_{2}, E_{2}\right)$ be graphs. The product of $G_{1}$ and $G_{2}$ is the graph $G_{1} \times G_{2}$ which has vertex set $V_{1} \times V_{2}$ and an edge between distinct vertices $\left(x_{1}, x_{2}\right)$ and $\left(y_{1}, y_{2}\right)$ if and only if $x_{i}=y_{i}$ or $\left\{x_{i}, y_{i}\right\} \in E_{i}$ for $i=1,2$. For $d \geqslant 1$ the graph power $G^{d}$ is the product of $G$ with itself $d$ times. The Shannon capacity of $G$ is

$$
c(G)=\sup _{d}\left(\alpha\left(G^{d}\right)\right)^{1 / d}
$$

where $\alpha(H)$ is the size of the largest independent set in graph $H$. This graph invariant was introduced by Shannon in 1956 and gives a measure of the optimal zero-error performance of an associated memoryless communication channel [19].

\footnotetext{
*Supported in part by NSF grant DMS 0401147.

${ }^{\dagger}$ Research supported by the Fund for the Promotion of Research at the Technion and by the P. and E. Nathan Research Fund.
} 
Determining the Shannon capacity of an arbitrary graph is a notoriously hard problem. Shannon himself gave an upper bound, which may be stated in graph thoretic notation as $c(G) \leqslant \alpha^{*}(G)$, where $\alpha^{*}(G)$ is the fractional vertex packing number. (The latter is a natural linear programming relaxation of the independence number; namely, $\alpha^{*}(G)$ is the maximum, over all assignments of non-negative real weights to the vertices of $G$ with the property that the sum of weights over any clique is at most 1 , of the sum of weights of the vertices of $G$.) This upper bound was discussed in detail in [18]. A better upper bound $c(G) \leqslant \theta(G)$ is given by the Lovász theta function [16]. Another upper bound, which is not comparable with the former two, was given by Haemers [11]. (For a survey of zero-error information theory see [15]. For recent progress on the sums of disjoint channels using linear algebraic methods see [1], [2].)

Motivated in part by the problem of determining the Shannon capacities of graphs, Berge introduced the notion of a perfect graph [4]. A graph $G$ is perfect if $\omega(H)=\chi(H)$ for every induced subgraph $H$ of $G$, where $\omega(H)$ is the size of the largest clique in $H$ and $\chi(H)$ is the chromatic number of $H$. Perfect graphs are known to satisfy $c(G)=\alpha(G)$. Berge conjectured that a graph is perfect if and only if it does not contain an odd cycle of length five or more, or the complement of such a graph, as an induced subgraph. This long standing conjecture, known as the strong perfect graph conjecture, was recently proved by Chudnovsky, Robertson, Seymour and Thomas [8] (for an overview of the proof, see [10]). It follows from this result that the odd cycles of length five or more and their complements are the minimal graphs for which the determination of the Shannon capacity is nontrivial. The best known upper bounds on the Shannon capacities of these graphs are given by the Lovász theta function. This upper bound suffices to establish the Shannon capacity of $C_{5}$, which is self complementary: $c\left(C_{5}\right)=c\left(\bar{C}_{5}\right)=\sqrt{5}$. However, the Shannon capacities of odd cycles and the complements of odd cycles on seven or more vertices remain unknown.

In this paper we focus on odd cycles (for recent progress on the complements of odd cycles see [6]) and consider the following question: For fixed values of $d$ and $\ell$ what is $\alpha\left(C_{2 \ell+1}^{d}\right)$ ? This question was first addressed some time ago by Baumert, McEliece, Rodemich, Rumsey, Stanley and Taylor [3] and Hales [12]. In [3] it is noted that this question is equivalent to the following natural question: How many $d$-dimensional cubes of side length 2 can we pack into a $d$-dimensional torus of side length $2 \ell+1$ ?

While there are a number of constructions (see [3], [5], [9], [12]) that give lower bounds on $\alpha\left(C_{2 \ell+1}^{d}\right)$, there are essentially no upper bounds that take into account the special structure of odd cycles. We get an upper bound on $\alpha\left(C_{2 \ell+1}^{d}\right)$ by appealing to either the Lovász theta function,

$$
\alpha\left(G^{d}\right) \leqslant \vartheta(G)^{d}
$$

or the well-known inequality (proved below in Section 2):

$$
\alpha(G \times H) \leqslant \alpha(G) \alpha^{*}(H) .
$$

The only choice of parameters $d, \ell$ for which we have an upper bound on $\alpha\left(C_{2 \ell+1}^{d}\right)$ that is better than that given by (1) and (2) (other than two specific small examples) is the case $d \geqslant 3$ and $\ell \equiv 2^{d-2} \bmod 2^{d-1}$. Here Baumert et al. [3] proved that the independence 
number is at most the bound given by (2) minus 1 . On the other hand, there are values of parameters $d, \ell$ where (1) or (2) give the truth:

$$
\begin{gathered}
\alpha\left(C_{5}^{2 j}\right)=5^{j}=\vartheta\left(C_{5}\right)^{2 j} \\
\alpha\left(C_{k 2^{d}+1}^{d}\right)=k\left(k 2^{d}+1\right)^{d-1}=2^{d-1} k\left(\frac{k 2^{d}+1}{2}\right)^{d-1}=\alpha\left(C_{k 2^{d}+1}\right) \alpha^{*}\left(C_{k 2^{d}+1}^{d-1}\right) \\
\alpha\left(C_{k 2^{d}+3}^{d}\right)=\frac{k\left(k 2^{d}+3\right)^{d}+1}{k 2^{d}+1}=\left\lfloor\left(\frac{2 k\left(k 2^{d}+3\right)^{d-1}+1}{k 2^{d}+1}\right)\left(\frac{k 2^{d}+3}{2}\right)\right\rfloor \\
=\left\lfloor\alpha\left(C_{k 2^{d}+3}^{d-1}\right) \alpha^{*}\left(C_{k 2^{d}+3}\right)\right\rfloor
\end{gathered}
$$

Equation (3) was established in the celebrated paper of Lovász [16]. Hales [12] and Baumert et al. [3] independently established (4), and Baumert et al. [3] proved (5).

Given this state of affairs, the first interesting case is $\alpha\left(C_{8 n+5}^{3}\right)$. Setting

$$
t_{n}=(8 n+5) \frac{(2 n+1)(8 n+5)-1}{2},
$$

we can summarize the current state of our understanding as follows:

$$
t_{n} \leqslant \alpha\left(C_{8 n+5}^{3}\right) \leqslant t_{n}+4 n+1 .
$$

The lower bound is given by construction [3],[5] (for the sake of completeness one of these constructions is reproduced in Subsection 1.1 below). The upper bound is one less than the bound given by (2) (as established in [3]). (For small values of $n$ the Lovász theta function gives a slightly better upper bound.) Baumert et al. [3] conjectured that $\alpha\left(C_{8 n+5}^{3}\right)=t_{n}$ for all $n$.

Now, we are ready to state our main results.

Theorem 1. If $8 n+5$ is prime then $\alpha\left(C_{8 n+5}^{3}\right)=t_{n}$.

This settles the above conjecture for an infinite sequence of cycle lengths. In particular, it yields $\alpha\left(C_{13}^{3}\right)=247$. (Codenotti et al. [9] used a computer search to establish the best previously known upper bound of 251 for this value.) Natarajan [17] has recently settled the conjecture for another infinite sequence; namely, he proved that if $2 n+1$ is prime then $\alpha\left(C_{8 n+5}^{3}\right)=t_{n}$. For general $n$, we obtain an upper bound which differs from the conjectured value by 2 .

Theorem 2. For all n,

$$
\alpha\left(C_{8 n+5}^{3}\right) \leqslant t_{n}+2 .
$$

Our proofs are based on a dimension-reduction technique, which may be briefly explained as follows. We consider the two-dimensional slices of $C_{8 n+5}^{3}$, which we call 'planes'. Each of them consists of the vertices that have, in a given coordinate (the 'direction' of the plane), one of two consecutive values, and arbitrary values in the other two coordinates. Given an independent set $S$ in $C_{8 n+5}^{3}$, its intersection with each plane projects onto an 
independent set in $C_{8 n+5}^{2}$. If $S$ contains more than $t_{n}$ vertices, then a calculation shows that in each direction there must be at least two planes for which this projection yields a maximum independent set in $C_{8 n+5}^{2}$, and for most planes it yields independent sets of size at least $\alpha\left(C_{8 n+5}^{2}\right)-1$. Now, the structure of maximum independent sets in $C_{8 n+5}^{2}$ is well understood (due to [3]). Our proof of Theorem 1 consists of showing that, when $8 n+5$ is prime, six planes with this structure - two in each of the three directions cannot fit together. This is no longer true when $8 n+5$ is composite, and therefore we consider in the proof of Theorem 2 also those planes with $\alpha\left(C_{8 n+5}^{2}\right)-1$ vertices of $S$. We have obtained a full characterization of these almost maximum independent sets. This may be viewed as a stability result, essentially showing that all almost maximum independent sets are slightly modified maximum ones. The proof of Theorem 2 then consists of showing that many planes in every direction, each of them having the structure of a maximum independent set or a slight modification of it, cannot fit together. Because the statement of the above-mentioned stability result is complicated and its proof is tedious, they do not appear in this version of the paper; only the relevant consequences for the proof of Theorem 2 are stated. The interested reader is referred to the original, much longer version of the paper (available from the first author's home page). We emphasize that the proof of Theorem 1 does not require this stability result; the proof of Theorem 1 is short and completely contained herein. For other applications of stability methods in extremal combinatorics, in particular in the context of hypergraph Turán theory, see for example [13], [14], [20].

Although our main results only deal with three dimensions and cycles of length $5(\bmod 8)$, we believe that the methods developed here are of interest for the more general problem of giving upper bounds on the independence numbers of powers of odd cycles. Good upper bounds for arbitrary powers of odd cycles (at least of certain lengths) would lead to improved upper bounds on, or even a determination of, the Shannon capacities of these graphs. This is the ultimate goal, yet it looks pretentious at this stage. While the dimension-reduction technique described above is available in all dimensions, the structural information about the lower-dimensional objects is not (it could possibly be achieved inductively). For many cycle lengths, there are not even good-looking constructions, the optimality of which one could hope to prove; such is the case with $C_{7}$. Still, we have had some success applying similar methods to higher powers. In [7] we gave a complete classification of all maximum independent sets in powers of odd cycles of the form $C_{k 2^{d}+1}^{d}$. In other (unpublished) work, we used stability results for large independent sets in $C_{9}^{2}$ and $C_{9}^{3}$ to establish the upper bound $\alpha\left(C_{9}^{4}\right) \leqslant 350$. As $\alpha\left(C_{9}^{3}\right)=81$ by (4), this shows that $\left(\alpha\left(C_{9}^{4}\right)\right)^{1 / 4}<\left(\alpha\left(C_{9}^{3}\right)\right)^{1 / 3}$, and thus offers some support for our conjecture that the Shannon capacity of $C_{9}$ is attained in the third power.

The remainder of this paper is organized as follows. In Subsection 1.1 we give a construction that achieves the lower bound in (6). In Section 2 we introduce some preliminary facts, including the classification of maximum independent sets in $C_{4 m+1}^{2}$. Theorem 1 is proved in Section 3. The proof of Theorem 2 is described in Section 4, omitting the details of the stability part (as explained above). 


\subsection{Construction}

The construction we give here is taken from [5].

We assume $n \geqslant 1$, and identify $C_{8 n+5}$ and $\mathbb{Z}_{8 n+5}$ in the natural way. Define

$$
J=\left\{(x, y, z) \in \mathbb{Z}_{8 n+5}^{3}: 4 x+2 y+z=0\right\},
$$

where the operation is taken over $\mathbb{Z}_{8 n+5}$. Note that $J$ is an independent set as the difference of a pair of distinct elements of $J$ is in $J$ and therefore not in the set $\{-1,0,1\}^{3}$. We will make use of the stronger property that no pair of distinct elements of $J$ have difference in the set $[-2 n, 2 n] \times\{-1,0,1\}^{2}$. This property allows us to form an independent set by replacing each element $a$ of $J$ with a collection of vertices 'centered' at $a$. Let $e_{1}$ be the first standard basis vector and for $i \geqslant 1$ let

$$
P_{i}=\left\{(-i+1) e_{1},(-i+3) e_{1}, \ldots,(i-3) e_{1},(i-1) e_{1}\right\}
$$

Note that $\left|P_{i}\right|=i$ and that $P_{i}$ is an independent set. Define the parity of $(x, y, z) \in J$ to be the parity of $z$. Our independent set of size $t_{n}$ is the following:

$$
I=\left(\bigcup_{a \in J: a \text { is even }} a+P_{n}\right) \cup\left(\bigcup_{a \in J: a \text { is odd }} a+P_{n+1}\right) .
$$

Since no pair of distinct elements of $J$ have difference in $[-2 n, 2 n] \times\{-1,0,1\}^{2}$, if $a, b$ are distinct elements of $J$ then no vertex in $a+P_{n}$ is adjacent to a vertex in $b+P_{n}$ or in $b+P_{n+1}$. So, we can restrict our attention to odd $a, b$ with the same third coordinate and second coordinate differing by 1 . Such pairs of vertices differ by $4 n+2$ in the first coordinate.

\section{Preliminaries}

\subsection{Proof of inequality (2)}

We consider an independent set $S$ in the product $G \times H$ of graphs $G$ and $H$. To every vertex $y$ of $H$ we assign the weight $w(y)=|S \cap(G \times\{y\})| / \alpha(G)$. If $K$ is a clique in $H$ then the first coordinates of the vertices in $S \cap(G \times K)$ must form an independent set in $G$; therefore we have $\sum_{y \in K} w(y) \leqslant 1$. Hence this is a fractional vertex packing, and thus its total weight $|S| / \alpha(G)$ is a lower bound on $\alpha^{*}(H)$. Inequality (2) follows.

Although this inequality is well known, we included the proof because the consideration of sets of the form $G \times K$, where $K$ is a clique in $H$, is the essence of our dimensionreduction technique (see the concept of a slice, which we introduce below).

\subsection{Notational conventions}

Throughout the paper we discuss products and powers of odd cycles, in many cases restricting attention to cycle lengths having certain residues modulo 4 or 8 . In an attempt 
to reduce confusion, we use $\ell$ to write an arbitrary odd length as $2 \ell+1$, we use $m$ when we care about the residue modulo 4 , e.g., $4 m+1$, and we use $n$ when we care about the residue modulo 8 , e.g., $8 n+5$.

We identify the vertices of $C_{2 \ell+1}$ with the elements of $\mathbb{Z}_{2 \ell+1}$ throughout. Unless otherwise noted, operations on vertices are taken over this ring.

We use the standard notation $[d]=\{1, \ldots, d\}$.

\subsection{Slices}

Consider the graph $G=\prod_{i=1}^{d} C_{2 \ell_{i}+1}$. Given a set $I \subseteq[d]$ and a vector $\mathbf{x} \in \prod_{i \in I} \mathbb{Z}_{2 \ell_{i}+1}$, the slice of $G$ given by $I=\left\{s_{1}, \ldots, s_{k}\right\}$ and $\mathbf{x}=\left(x_{1}, \ldots, x_{k}\right)$ is the set of vertices

$$
\left\{\left(v_{1}, \ldots, v_{d}\right) \in G: v_{s_{i}} \in\left\{x_{i}, x_{i}+1\right\} \text { for } i=1, \ldots, k\right\} .
$$

Note that when we drop the coordinates in $I$ this slice projects onto the graph $\prod_{i \notin I} C_{2 \ell_{i}+1}$. Furthermore, if $S$ is an independent set in $G$ then $S$ intersected with the slice maps onto an independent set in $\prod_{i \notin I} C_{2 \ell_{i}+1}$ under this projection. The dimension of the slice given by $I$ and $\mathbf{x}$ is $d-|I|$. Here we will work extensively with two special cases of slices.

Definition 3. Consider the graph $C_{2 \ell+1}^{3}$. Let $d \in[3]$ and $y \in \mathbb{Z}_{2 \ell+1}$. We define the dual plane

$$
P_{y, d}=\left\{\left(x_{1}, x_{2}, x_{3}\right) \in \mathbb{Z}_{2 \ell+1}^{3}: x_{d} \in\{y, y+1\}\right\} .
$$

If $S \subset C_{2 \ell+1}^{3}$ is an independent set then we set $S_{y, d}=S \cap P_{y, d}$. Note that we can view $S_{y, d}$ as an independent set in $C_{2 \ell+1}^{2}$ by simply dropping coordinate $d$.

Definition 4. Consider the graph $G=C_{2 \ell_{1}+1} \times C_{2 \ell_{2}+1}$ and $S$ an independent set in $G$. The rth dual row is the set of all $(x, y) \in S$ so that $y \in\{r, r+1\}$. Similarly, the cth dual column is the set of all $(x, y) \in S$ so that $x \in\{c, c+1\}$. A dual line is a dual row or a dual column.

\subsection{Holes}

The property of being an independent set may be defined by the system of constraints requiring that at most one vertex be picked from every maximal (with respect to inclusion) clique. In describing a particular independent set, it is useful to note which of these constraints are satisfied as equalities and which of them are not. In the following definition we focus on the latter.

Definition 5. Let $G$ be a graph, and let $S$ be an independent set in $G$. A set of vertices $K$ is a hole of $S$ if $K$ is a maximal clique in $G$ and $K \cap S=\emptyset$. We let $H(S)$ denote the set of holes of the independent set $S$. In $G=\prod_{i=1}^{d} C_{2 \ell_{i}+1}$ with $\ell_{i} \geqslant 2$, the maximal cliques are precisely the maximum cliques, namely they are of the form $K_{v}:=\{v\}+\{0,1\}^{d}$. By a slight abuse of terminology, we say that $v$ is a hole if $K_{v}$ is. (In other words, a hole is a d-dimensional cube, but by convention, we specify it using the coordinate values of its lower-leftmost vertex.) 
Note that if $S_{1}$ and $S_{2}$ are independent sets in a product of odd cycles then $H\left(S_{1}\right)=H\left(S_{2}\right)$ if and only if $S_{1}=S_{2}$. (To see this, consider the set of 1-dimensional slices through a clique $K_{v}$ that is not a hole. The holes in these slices determine the location of the one vertex in $S \cap K_{v}$ by parity.) Also note that the holes in a slice of an independent set $S$ correspond to holes in $H(S)$.

The following facts concerning the parity of the number of holes in certain segments are easy to check (using the observation that every element of $S$ in the interior of the segment accounts for exactly two non-holes).

Lemma 6. Let $S$ be an independent set in $C_{2 \ell_{1}+1} \times C_{2 \ell_{2}+1}$.

(i) If $\left(x_{1}, y_{1}\right),\left(x_{2}, y_{2}\right) \in S$ where $x_{1}<x_{2}$ and $y_{2} \in\left\{y_{1}, y_{1}+1\right\}$ then

$$
\left|\left\{\left(x, y_{1}\right) \in H(S): x \in\left[x_{1}, x_{2}\right]\right\}\right| \equiv x_{2}-x_{1} \quad \bmod 2 .
$$

(ii) If $\left(x_{1}, y_{1}\right) \in H(S)$ and $\left(x_{2}, y_{2}\right) \in S$ where $x_{1}<x_{2}$ and $y_{2} \in\left\{y_{1}, y_{1}+1\right\}$ then

$$
\left|\left\{\left(x, y_{1}\right) \in H(S): x \in\left(x_{1}, x_{2}\right]\right\}\right| \equiv x_{2}-x_{1} \quad \bmod 2 .
$$

By a similar argument, if $S$ is an independent set in a product of two odd cycles then every dual line has an odd number of holes; in particular, every line has a hole. If the size of the independent set is near the maximum then most rows and most columns will have only one hole - a few dual lines may contain more than one hole.

Definition 7. Let $S$ be an independent set in $C_{2 \ell_{1}+1} \times C_{2 \ell_{2}+1}$. A dual line is $k$-bad (or has badness $k$ ) in $S$ if it has $2 k+1$ holes in $S$. A dual line is full if it is 0-bad.

The following simple lemma will help prove that certain independent sets are significantly below the maximum possible size:

Lemma 8. Let $S$ be an independent set in $C_{2 \ell_{1}+1} \times C_{2 \ell_{2}+1}$, and suppose that $(0,0)$ and $(2 k+1,1)$ are holes in $S$. Suppose further that dual rows 0 and 1 are both full. Then $S$ contains at most $k$ vertices in row 1 . Consequently, $|S| \leqslant \ell_{1} \ell_{2}+k$.

Proof. Suppose that $S$ contains $(x, 1)$ for some $x \in\left[2 k+3,2 \ell_{1}\right]$. If $x$ is odd, Lemma 6(ii) applied to dual row 0 and the hole $(0,0)$ implies another hole in that row. If $x$ is even, Lemma 6(ii) applied to dual row 1 and the hole $(2 k+1,1)$ implies another hole in that row. As we assume that both rows are full, we get a contradiction in either case, so $S$ contains no such vertices. Therefore, $|\{x:(x, 1) \in S\}| \leqslant k$. The final statement follows by noticing that each dual row contains at most $\ell_{1}$ vertices from $S$, and applying this observation to even-numbered dual rows from 2 through $2 \ell_{2}$. 


\subsection{Maximum independent sets in $C_{4 m+1}^{2}$}

Baumert et al. [3] characterized the maximum independent sets in $C_{4 m+1}^{2}$. Their characterization may be stated in terms of the hole structure as follows.

Lemma 9 (Baumert et al.). Let $S$ be a maximum independent set in $C_{4 m+1}^{2}$. Then $|S|=m(4 m+1)$. Furthermore, there exist $\alpha>0$ such that $\alpha \mid m, \epsilon \in\{ \pm 1\}$ and $\left(t_{1}, t_{2}\right) \in \mathbb{Z}_{4 m+1}^{2}$ such that

$$
H(S)=\left(t_{1}, t_{2}\right)+\langle(2 \epsilon \alpha, 1)\rangle,
$$

where $\langle(2 \epsilon \alpha, 1)\rangle$ is the cyclic subgroup generated by $(2 \epsilon \alpha, 1)$.

Proof. For the reader's convenience, we sketch the proof. Suppose that $S \subset C_{4 m+1}^{2}$ is an independent set of size $m(4 m+1)$. Then it has exactly one hole in every dual line. An inclusion-maximal subset of $S$ of the form $\{(x, y),(x+2, y), \ldots,(x+2 j, y)\}$ will be called a thread of length $j+1$ in row $y$. The intersection of $S$ with any given row is the union of some number of threads.

Now, assume without loss of generality that the holes in dual rows 0 and 1 are at $(0,0)$ and $(2 k, 1)$ respectively, where $k>0$. Let us compare the thread structure of rows 0 and 2 . It follows from Lemma 6 that $\{(2,0),(4,0), \ldots,(2 k, 0)\}$ and $\{(1,2),(3,2), \ldots,(2 k-1,2)\}$ are threads of length $k$ in these two rows, and the other threads in these two rows occupy the same horizontal positions. Thus, the thread structure of row 2 is obtained from that of row 0 by a unit shift of one thread. Now we consider the sequence of thread structures of rows $0,2,4, \ldots, 4 m, 1,3, \ldots, 4 m-1,0$. By the above, each thread structure in this sequence is obtained from its predecessor by a unit shift of one thread. Given that we return to the original thread structure after $4 m+1$ steps, it must be the case that in the whole process each thread is shifted to the position of the next thread (in some cyclic order on the threads). It follows that all threads in all rows have the same length, say $\alpha$, and all rows have the same number of threads, say $\beta$. A calculation gives $\alpha \beta=m$, and hence $\alpha \mid m$. We get the statement of the lemma by observing that the $k$ we started with must be equal to $\alpha$, and it must be the same for any choice of two adjacent dual rows.

Note that the independent set generated by $(2 \epsilon \alpha, 1)$ is also generated by $(1,-2 \epsilon \beta)$ where $\alpha \beta=m$. So, the number of distinct (up to isomorphism) maximum independent sets in $C_{4 m+1}^{2}$ equals the number of factorizations $m=\alpha \beta$ where $\alpha \leqslant \beta$. The class of a maximum independent set $S$ in $C_{4 m+1}^{2}$ is the positive integer $\alpha \leqslant \sqrt{m}$ such that $S$ is generated by $( \pm 2 \alpha, 1)$ or $(1, \pm 2 \alpha)$. The orientation of $S$ is determined by the coordinate in which this generator has a 1. So, if $\alpha \mid m, \alpha \leqslant \sqrt{m}$ and $\gamma \in\{1,2\}$ then there are (up to translations) two maximum independent sets in $C_{4 m+1}^{2}$ of class $\alpha$ and orientation $\gamma$. An example of a maximum independent set in $C_{25}^{2}$ of class 2 and orientation 1 is given in Figure 1.

\section{Proof of Theorem 1}

Let $S$ be an independent set in $C_{8 n+5}^{3}$. The plane $P_{x, d}$ is full if $S_{x, d}$ is a maximum independent set in $C_{8 n+5}^{2}$. We begin with a simple counting observation. 


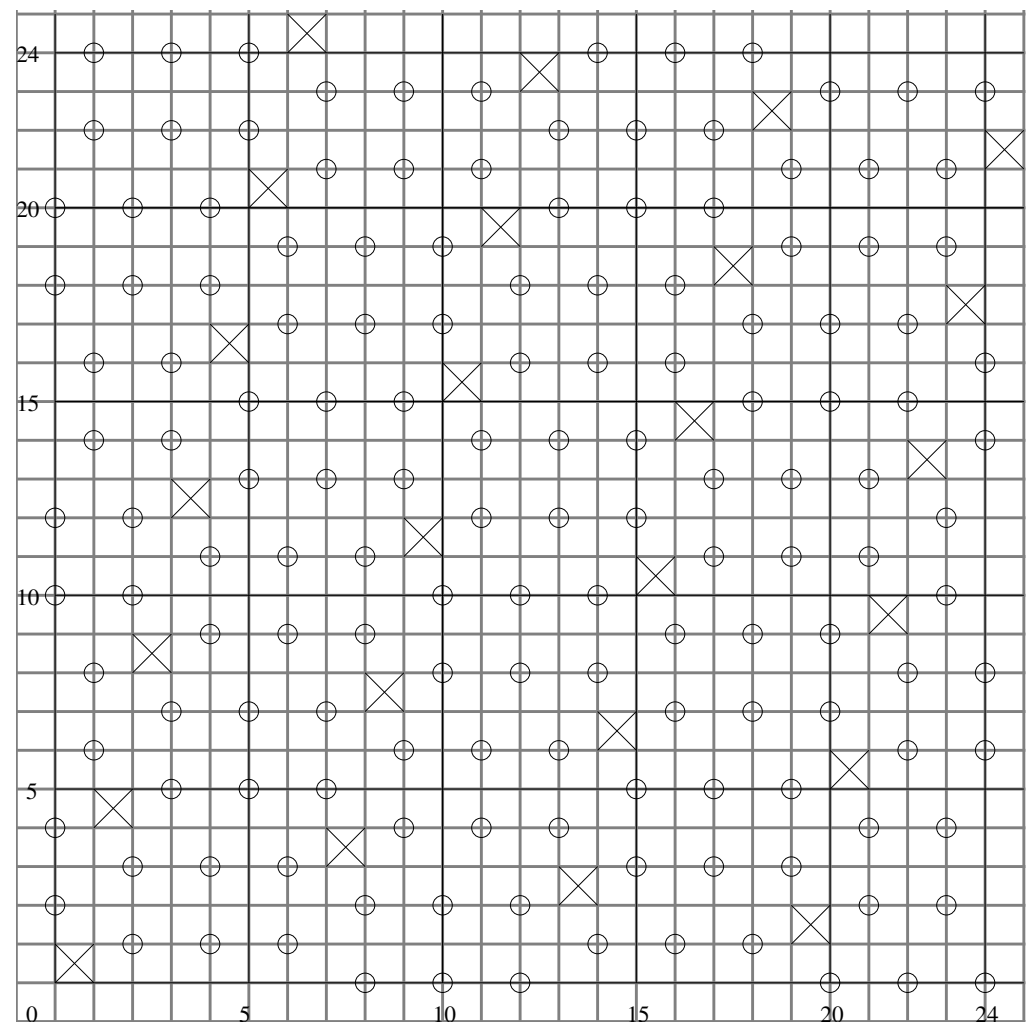

Figure 1: A maximum independent set in $C_{25}^{2}$ of class 2. The holes are marked with x's.

Lemma 10. Let $S$ be an independent set in $C_{8 n+5}^{3}$ so that $|S|=t_{n}+k$. Then, for each $d \in[3]$, there are at least $2 k$ distinct values of $x$ so that $P_{x, d}$ is full.

In other words, if the size of the independent set exceeds the best known lower bound then there are (at least two) full planes 'in each direction.' Since each hole in $C_{8 n+5}^{3}$ is a hole in three dual planes, we can use Lemma 9 to relate the structure of intersecting full planes. To express this, we introduce notation that takes advantage of Lemma 9. For each $i \in[3]$, let $e_{i}$ be the $i^{\text {th }}$ standard basis vector.

Definition 11. Let $S$ be an independent set in $C_{8 n+5}^{3}$, and suppose that $P_{x, d}$ is full. Let $\{i, j\}=[3] \backslash\{d\}$. Then $\Delta_{x, d, i}$ is defined to be the unique even integer (possibly negative) such that if $v=\left(x_{1}, x_{2}, x_{3}\right)$ is a hole with $x_{d}=x$ then $v+e_{i}+\Delta_{x, d, i} e_{j}$ is also a hole.

Thus, $\Delta_{x, d, i}$ corresponds to $2 \epsilon \alpha$ in the notation of Lemma 9. Note that $\Delta_{x, d, i}$ is invertible in $\mathbb{Z}_{8 n+5}$ and $\Delta_{x, d, j}=\Delta_{x, d, i}^{-1}$.

Let $S$ be an independent set in $C_{8 n+5}^{3}$ such that $P_{0, d}$ is full for each $d \in[3]$ and let $(x, 0,0)$ be a hole in $S$. Then, it follows from Definition 11 that we have

$$
\begin{aligned}
& (x, 0,0) \in H(S) \Rightarrow\left(0,-\Delta_{0,3,1} x, 0\right) \in H(S) \Rightarrow \\
& \quad\left(0,0,-\Delta_{0,1,2}\left(-\Delta_{0,3,1} x\right)\right) \in H(S) \Rightarrow\left(-\Delta_{0,2,3}\left(-\Delta_{0,1,2}\left(-\Delta_{0,3,1} x\right)\right), 0,0\right) \in H(S) .
\end{aligned}
$$


Since $P_{0,2}$ is full, $(x, 0,0)$ is the only hole in row 0 of $S_{0,2}$, implying $x=-\Delta_{0,2,3} \Delta_{0,1,2} \Delta_{0,3,1} x$. We have proved the following.

Lemma 12. If $S$ is an independent set in $C_{8 n+5}^{3}, P_{0, d}$ is full for each $d \in[3]$ and $(x, 0,0)$ is a hole in $S$ then

$$
-\Delta_{0,1,2} \Delta_{0,2,3} \Delta_{0,3,1} x=x .
$$

In some very special cases this observation alone is enough to determine the independence number of $C_{8 n+5}^{3}$.

Suppose, for example, that $8 n+5$ and $2 n+1$ are both prime (e.g., $n=1,3,6$ ) and that $S$ is an independent set in $C_{8 n+5}^{3}$ such that $|S|>t_{n}$. By Lemma 10, without loss of generality $P_{0, d}$ is full for all $d \in[3]$ and $(x, 0,0)$ is a hole for some $x \neq 0$ (here we use the existence of at least two full planes for $d=1$ ). Since $2 n+1$ is prime, we also have $\Delta_{0,1,2}, \Delta_{0,2,3}, \Delta_{0,3,1} \in\{ \pm 2, \pm(4 n+2)\}$. Then Lemma 12 implies

$$
8 x= \pm x \quad \text { or } \quad 2 x= \pm x .
$$

Since $8 n+5$ is prime, $x$ is coprime to $8 n+5$, and we have a contradiction in either case.

When $2 n+1$ is not prime, we can no longer restrict $\Delta_{x, i, j}$ to such a small set of values. However, if $8 n+5$ is prime, we need only ensure that the coefficient of $x$ in (7) is not 1 . It turns out that this is the case.

Lemma 13. If $x_{i}$ is an even divisor of $4 n+2$ for each $i \in[3]$ then $x_{1} x_{2} x_{3} \not \equiv \pm 1 \bmod (8 n+$ 5).

Proof. Assume for the sake of contradiction that $x_{1} x_{2} x_{3} \equiv \pm 1 \bmod (8 n+5)$. Without loss of generality, $x_{i}>0$ for each $i \in[3]$. Let $y_{i}=(8 n+4) / x_{i}$ for $i \in[3]$. Then $y_{i}=-\left(x_{i}\right)^{-1}$ in the ring $\mathbb{Z}_{8 n+5}$, for each $i \in[3]$. Furthermore, for each $i \in[3], y_{i}$ is also an even divisor of $4 n+2$. Therefore, for any distinct $i, j, k \in[3]$, we have:

$$
\begin{aligned}
x_{i} x_{j} & \equiv \pm y_{k} \bmod (8 n+5), \\
x_{k} & \equiv \pm y_{i} y_{j} \bmod (8 n+5) .
\end{aligned}
$$

For each $i$, either $x_{i} \leqslant \sqrt{8 n+5}$ or $y_{i} \leqslant \sqrt{8 n+5}$, so either two of the $x$ 's or two of the $y$ 's are at most $\sqrt{8 n+5}$. If two of the $x$ 's (say, $x_{i}$ and $x_{j}$ ) are at most $\sqrt{8 n+5}$ we have either $x_{i} x_{j}=y_{k}$ or $x_{i} x_{j}=(8 n+5)-y_{k}$. The former case yields a contradiction because $x_{i} x_{j}$, being the product of two even numbers, is a multiple of four, whereas $y_{k}$, dividing $4 n+2$, is not a multiple of four. The latter case yields a contradiction because $(8 n+5)-y_{k}$ is odd, and $x_{i} x_{j}$ is even. The case where two of the $y$ 's are at most $\sqrt{8 n+5}$ is handled similarly, using (10).

Corollary 14. Let $S$ be an independent set in $C_{8 n+5}^{3}$, and assume that $P_{0, d}$ is full for each $d \in[3]$, and $(x, 0,0)$ is a hole such that $x \neq 0$. Then $\operatorname{gcd}(x, 8 n+5)>1$.

Proof. Lemma 12 implies that $x\left(1+\Delta_{0,1,2} \Delta_{0,2,3} \Delta_{0,3,1}\right)=0$. Lemma 13 implies that the second factor is not 0 , thus requiring that the first factor be a zero divisor in $\mathbb{Z}_{8 n+5}$.

Note that Theorem 1 follows from Lemma 10 and Corollary 14. 


\section{Proof of Theorem 2}

So far, our techniques only utilize the positions of holes in full planes, and operate by showing that there is no way to place these holes in a manner consistent with Lemma 9. These techniques alone are insufficient when $8 n+5$ is not prime - for example, in $C_{21}^{3}$ it is possible, for each $d \in[3]$, to have two values of $x$ so that $P_{x, d}$ is full, and place the holes in all of the full $P_{x, d}$ in a seemingly consistent manner. To prove Theorem 2 , then, we will look also at planes that are just short of being full. It turns out that the structure of such planes is essentially governed by Lemma 9 as well, with certain types of modifications allowed. Moreover, when such a plane lies right next to a full plane, then (subject to certain provisions) the maximum independent sets underlying the two of them must be of the same class and orientation, which in turn constrain the class and orientation of full planes in the other directions. Very roughly speaking, these considerations will allow us to derive a contradiction similar to that obtained in the previous section, without assuming the primality of $8 n+5$, but we will need a larger number of full planes in each direction (hence the +2 in the upper bound that we establish).

Motivated by this discussion, we introduce the following terminology. Let $S$ be an independent set in $C_{8 n+5}^{3}$. To each full plane $P_{x, d}$ we assign a class and orientation, namely those of the maximum independent set $S_{x, d}$. The plane $P_{x, d}$ is almost full if $\left|S_{x, d}\right|=\alpha\left(C_{8 n+5}^{2}\right)-1$. The plane $P_{x, d}$ is good if $P_{x, d}$ is full and $P_{x-1, d}$ or $P_{x+1, d}$ is full or almost full; it is solid if $P_{x, d}$ is full and $P_{x-1, d}$ and $P_{x+1, d}$ are both full or almost full.

In the proof of Theorem 2, we consider a large independent set $S$ in $C_{8 n+5}^{3}$, and gradually establish facts about the number of good planes in each direction and their classes, leading to a contradiction in the end. To facilitate the understanding of the structure of the proof, we break it into several steps. The title of each subsection states, rather informally, what is established in that step.

\subsection{There are many good planes in each direction}

Here we prove a refinement of Lemma 10, guaranteeing planes that are not only full but actually good, and showing that their number must be even larger in certain circumstances (namely, if some planes contain independent sets that are significantly smaller than the maximum). The following function measures the size of the independent sets in the various planes, normalized so that almost full planes get the value zero. For $d \in[3]$ define $\delta_{S, d}: \mathbb{Z}_{8 n+5} \rightarrow \mathbb{Z}$ by

$$
\delta_{d}(x)=\delta_{S, d}(x)=\left|S_{x, d}\right|-\alpha\left(C_{8 n+5}^{2}\right)+1 .
$$

Lemma 15. Let $S$ be an independent set in $C_{8 n+5}^{3}$ such that $|S|=t_{n}+k$, and let $d \in[3]$. Then the number of good planes $P_{x, d}$ is at least

$$
2 k+\sum_{x: \delta_{d}(x) \leqslant-2}\left(-\delta_{d}(x)-1\right)+1_{\exists x \text { such that } \delta_{d}(x)<0} .
$$

Before proving this lemma we state some definitions that will also be used below. Let a $\delta_{d}$-streak be a maximal interval $I$ in $\mathbb{Z}_{8 n+5}$ such that $\delta_{d}(x) \neq 0$ for all $x \in I$, no 
two consecutive $x, y \in I$ have $\delta_{d}(x)=\delta_{d}(y)=1$, and no two consecutive $x, y \in I$ have $\delta_{d}(x), \delta_{d}(y)<0$. A streak $I$ is a singleton if $|I|=1$ and $\delta_{d}(x)=1$ for $x \in I$, and a streak $I=[a, b]$ is positive if $|I|>1$ and $\delta_{d}(a)=\delta_{d}(b)=1$. Note that the set of streaks forms a partition of the set of $x \in \mathbb{Z}_{8 n+5}$ such that $\delta_{d}(x) \neq 0$. Each singleton streak corresponds to a solid plane. Each positive streak begins and ends with a good plane. Finally, note that streaks that are neither positive nor singletons may have good planes at one end.

Proof of Lemma 15. Let $s$ be the number of singleton streaks and $p$ be the number of positive streaks in the sequence $\delta_{d}(x)$. Since $\sum_{x \in \mathbb{Z}_{8 n+5}}\left|S_{x, d}\right|=2|S|$, we have

$$
2 k=\sum_{x \in \mathbb{Z}_{8 n+5}} \delta_{d}(x) \leqslant s+p+\sum_{x: \delta_{d}(x) \leqslant-2}\left(\delta_{d}(x)+1\right) .
$$

Note that the number of good planes is at least $s+2 p$. We get the statement of the lemma by noting that if there is an $x$ such that $\delta_{d}(x)<0$ then there is at least one streak that is not a singleton. This streak gives an additional good plane (beyond the accounting already presented).

Define

$$
\begin{aligned}
X=X_{S} & =\left\{x \in \mathbb{Z}_{8 n+5} \mid P_{x, 1} \text { is good }\right\}, \\
Y=Y_{S} & =\left\{y \in \mathbb{Z}_{8 n+5} \mid P_{y, 2} \text { is good }\right\}, \\
Z=Z_{S} & =\left\{z \in \mathbb{Z}_{8 n+5} \mid P_{z, 3} \text { is good }\right\} .
\end{aligned}
$$

Note that Lemma 15 implies that if $|S| \geqslant t_{n}+3$ then each of these sets has cardinality at least 6 .

\subsection{A good plane of class 1 implies a high ratio between the numbers of good planes in the other directions}

Supposing that there is a good plane of class 1, we apply here Lemma 8 to show that some planes in one of the other directions are significantly underused, and hence by Lemma 15 the number of good planes in that direction must be very large to compensate for that.

Lemma 16. Let $S$ be an independent set in $C_{8 n+5}^{3}$ such that $|S| \geqslant t_{n}+3$. If $P_{0,1}$ is good and $\Delta_{0,1,2}=4 n+2$ then

$$
|Y| \geqslant(n-1)(|Z|-4)+7 .
$$

Before proving Lemma 16, we make (as usual) some preliminary definitions and observations. Let $f_{S}^{+}, f_{S}^{-}: Z \rightarrow \mathbb{Z}_{8 n+5}$ be defined by

$$
\begin{aligned}
& f^{+}(z)=f_{S}^{+}(z)=-2 z+\Delta_{z, 3,1}, \\
& f^{-}(z)=f_{S}^{-}(z)=-2 z-\Delta_{z, 3,1} .
\end{aligned}
$$

Define $Y^{+}=Y_{S}^{+}$to be the range of $f_{S}^{+}$and $Y^{-}=Y_{S}^{-}$to be the range of $f_{S}^{-}$. Note that if $S$ is an independent set so that $P_{0,1}$ is full with $\Delta_{0,1,2}=4 n+2,(0,0,0)$ is a hole, and $z \in Z$ then $(0,-2 z, z),\left(1, f_{S}^{+}(z), z\right)$, and $\left(-1, f_{S}^{-}(z), z\right)$ are holes. 
Lemma 17. Suppose $P_{0,1}$ is full so that $\Delta_{0,1,2}=4 n+2,(0,0,0)$ is a hole, and $z \in Z$. If column 1 is full in $P_{f^{+}(z), 2}$ then:

$$
\begin{aligned}
\left|S_{f^{+}(z), 2}\right| & \leqslant(2 n+1)(8 n+4)+\frac{\left|\Delta_{z, 3,1}\right|-2}{4} \\
& =\alpha\left(C_{8 n+5}^{2}\right)-\left((2 n+1)-\left(\left|\Delta_{z, 3,1}\right|-2\right) / 4\right)
\end{aligned}
$$

In particular, $\left|S_{f^{+}(z), 2}\right| \leqslant \alpha\left(C_{8 n+5}^{2}\right)-(n+1)$. The analogous result holds for $S_{f^{-}(z), 2}$, if column -1 is full in $P_{f^{-}(z), 2}$.

Proof. As noted above, $\left(1, f^{+}(z), z\right)$ is a hole. On the other hand, by the definition of $f^{+}$and $\Delta_{0,1,2},\left(0, f^{+}(z), z-\Delta_{z, 3,1} / 2\right)$ is also a hole. Noting that $\left|\Delta_{z, 3,1} / 2\right|$ is odd, we then apply Lemma 8 to $P_{f^{+}(z), 2}$ to get the first inequality. The second one follows from $\left|\Delta_{z, 3,1}\right| \leqslant 4 n+2$. The proof for $S_{f^{-}(z), 2}$ is similar.

Lemma 18. Suppose $P_{0,1}$ is full so that $\Delta_{0,1,2}=4 n+2$, and $(0,0,0)$ is a hole. Let $y \in Y^{+}$, and suppose that column 1 is $k$-bad in $P_{y, 2}$. Then:

$$
\left|\left\{z \in Z \mid f^{+}(z)=y\right\}\right| \leqslant k+1
$$

A similar result holds for column -1, using $f^{-}$instead of $f^{+}$.

Proof. Let $z_{1}, z_{2} \in\left(f^{+}\right)^{-1}(\{y\})$. We have $-2 z_{1}+\Delta_{z_{1}, 3,1}=-2 z_{2}+\Delta_{z_{2}, 3,1}$, whence $z_{2}-z_{1}=$ $\left(\Delta_{z_{2}, 3,1}-\Delta_{z_{1}, 3,1}\right) / 2$. Now, $\Delta_{z_{2}, 3,1}$ and $\Delta_{z_{1}, 3,1}$ are both congruent to 2 modulo 4 , so it follows that $z_{2}-z_{1}$ is even. We order the elements $z_{1}, \ldots, z_{j}$ of $\left(f^{+}\right)^{-1}(\{y\})$ so that $z_{1} \leqslant \ldots \leqslant z_{j}$ and $z_{i+1}-z_{i}$ is even for $i=1, \ldots, j-1$. As noted above, $\left(1, y, z_{i}\right)$ is a hole for each $i=1, \ldots, j$. Consequently, by Lemma 6 , each interval $\left[z_{i}, z_{i+1}\right]$ in column 1 of $P_{y, 2}$ contains an additional hole. Since this column is $k$-bad, we get $2 j-1 \leqslant 2 k+1$, or $j \leqslant k+1$.

Proof of Lemma 16. We assume without loss of generality that $(0,0,0)$ is a hole and that $P_{1,1}$ is full or almost full. Since $P_{1,1}$ has at most two column badnesses, it follows from Lemma 18 that there are at most four $z \in Z$ such that $f^{+}(z)$ has the property that column 1 is bad in $P_{f^{+}(z), 2}$ (note that column 1 in $P_{f^{+}(z), 2}$ is column $f^{+}(z)$ in $\left.P_{1,1}\right)$. Let:

$$
Z^{*}:=\left\{z \in Z \mid \text { column } 1 \text { is full in } P_{f^{+}(z), 2}\right\} \text {. }
$$

We have $\left|Z^{*}\right| \geqslant|Z|-4$. Note that $f^{+}$, when restricted to $Z^{*}$, is one-to-one. Lemma 17 then implies

$$
\sum_{y: \delta_{2}(y) \leqslant-2}\left(-\delta_{2}(y)-1\right) \geqslant(n-1)\left|Z^{*}\right| \geqslant(n-1)(|Z|-4) .
$$

Appealing to Lemma 15, we have (11). 


\subsection{Handling the case $2 n+1$ prime}

If $2 n+1$ is prime and $S$ is an independent set in $C_{8 n+5}^{3}$ so that $|S| \geqslant t_{n}+3$, then every full plane is of class 1 and we get three instances of (11) (one in each 'direction'). Applying two of these in succession we have

$$
\max \{|X|,|Y|,|Z|\} \geqslant(n-1)(2 n+1)+7 \text {. }
$$

For $n \geqslant 5$ this is a contradiction, because the lower bound exceeds $8 n+5$. As we may assume, by Theorem 1 , that $8 n+5$ is composite, the only case left is $n=2$. In this case, the lower bound gives 12 good planes in some direction. Applying Corollary 14 with some fixed full planes in the other two directions and each of these 12 full planes, we get a contradiction because $\mathbb{Z}_{21}$ has only 9 elements that are divisible by 3 or 7 .

Thus, we may assume from now on that $2 n+1$ and $8 n+5$ are both composite. We will not use this assumption as such, but only its consequence that $n \geqslant 10$.

\subsection{Many good planes of classes $>1$ in different directions can fit together only if they are of the same class (with few exceptions)}

Now we turn to the good planes that are not of class 1. In order to explore the relationships among them, we define an auxiliary graph.

Definition 19. The tripartite directed graph $G_{S}$ is defined as follows. For each $d \in[3]$, let

$$
V_{d}:=\left\{x \in \mathbb{Z}_{8 n+5} \mid P_{x, d} \text { is good and not of class } 1\right\} \text {. }
$$

Set $V\left(G_{S}\right):=\bigcup_{d=1}^{3} V_{d}$. For any $x_{i}$ in $V_{i}$ and $x_{j}$ in $V_{j}$, with $i \neq j$, let $\left(x_{i}, x_{j}\right)$ be an arc if $\left|\Delta_{x_{i}, i, j}\right| \leqslant\left|\Delta_{x_{j}, j, i}\right|$.

The relevant feature of $G_{S}$ is that each node has a large out-degree to each of the other parts of the tripartition.

Lemma 20. Let $S$ be an independent set in $C_{8 n+5}^{3}, n \geqslant 8$, such that $|S| \geqslant t_{n}+3$. Let $x \in V_{i}$, and $j \in[3]$ with $j \neq i$. Then there are at most three $y \in V_{j}$ so that $(x, y)$ is not an arc in $G_{S}$. Furthermore, if there is an $A \subseteq V_{j}$ so that $|A|=3$ and $(x, y)$ is not an arc for all $y \in A$ then there is a $y \in A$ so that $y+1 \in A$.

The proof of Lemma 20 is based on a stability result for almost maximum independent sets in $C_{4 m+1}^{2}$ and some fine machinery applying this result to independent sets in $C_{8 n+5}^{3}$, $n \geqslant 8$, all developed in the original, long version of this paper. We refer the interested reader to that version, and give here only a very rough idea. We consider a full plane, say $P_{0,1}$, and an adjacent almost full plane, say $P_{1,1}$. The structure of $S_{1,1}$ must be a slight modification of the well-understood structure given in Lemma 9. The modification may significantly affect only a few dual columns of $P_{1,1}$; their numbers are collected in the exceptional set $A$ in the statement of the lemma. For any full plane $P_{y, 2}$ whose intersection 
with $P_{1,1}$ is not one of the exceptional columns, we have a good grasp of how its 0 and 1 columns look like (as they are in $P_{0,1}$ and $P_{1,1}$ ). This allows us to identify enough of the structure of $S_{y, 2}$ to deduce that $\left|\Delta_{y, 2,1}\right| \geqslant\left|\Delta_{0,1,2}\right|$. For these reconstruction arguments to work, we need to rule out planes of class 1 and small values of $n$.

Corollary 21. Let $S$ be an independent set in $C_{8 n+5}^{3}, n \geqslant 8$, such that $|S| \geqslant t_{n}+3$. Let $i, j \in[3], i \neq j$, satisfy $\left|V_{i}\right| \geqslant 4,\left|V_{j}\right| \geqslant 4$. Then there exist subsets $U_{i} \subseteq V_{i}$ and $U_{j} \subseteq V_{j}$ with $\left|U_{i}\right| \geqslant\left|V_{i}\right|-3,\left|U_{j}\right| \geqslant\left|V_{j}\right|-3$, so that the numbers $\left|\Delta_{x_{i}, i, j}\right|$ for $x_{i} \in U_{i}$ and $\left|\Delta_{x_{j}, j, i}\right|$ for $x_{j} \in U_{j}$ are all equal.

Proof. Consider the induced directed graph $G_{S}\left[V_{i} \cup V_{j}\right]$. Within any strongly connected component of it, the desired equalities hold by the definition of the arc set. One of these components, say $C$, has no arcs to the other components. It follows from Lemma 20 that $\left|C \cap V_{i}\right| \geqslant\left|V_{i}\right|-3$ and $\left|C \cap V_{j}\right| \geqslant\left|V_{j}\right|-3$.

\subsection{There is a good plane of class 1 in some direction}

Here we use the previous step to get a contradiction if there are at least 5 good planes of classes $>1$ in each direction. Since the total number of good planes in each direction is at least 6 , it will follow that there is a good plane of class 1 (in fact, two of them) in some direction.

Lemma 22. Let $S$ be an independent set in $C_{8 n+5}^{3}, n \geqslant 8$, such that $|S| \geqslant t_{n}+3$. Then there exists a $d \in[3]$ so that $\left|V_{d}\right| \leqslant 4$.

Proof. An observation that we use repeatedly below is that if plane $P_{x, d}$ is full and $\{i, j\}=$ $[3] \backslash\{d\}$ then $\Delta_{x, d, i} \Delta_{x, d, j}=-(8 n+4)$, and therefore $\left|\Delta_{x, d, i}\right| \leqslant \sqrt{8 n+4}$ if and only if $\left|\Delta_{x, d, j}\right| \geqslant \sqrt{8 n+4}$. We start with a technical claim similar to Lemma 13 .

Claim. If $P_{0, i}$ is full for each $i$ and $\Delta_{0, i, j} \in\{ \pm \sqrt{8 n+4}\}$ for all well-defined choices of $i$ and $j$, then $(0,0,0)$ is a hole.

Proof. By Lemma 12, it is sufficient to show that for all $n, \pm \sqrt{8 n+4}^{3} \pm 1$ is coprime to $8 n+5$. First, notice that $\sqrt{8 n+4}^{2}=8 n+4 \equiv-1 \bmod (8 n+5)$. Therefore, it is sufficient to show that $\pm \sqrt{8 n+4} \pm 1$ is coprime to $8 n+5$. We have $(\sqrt{8 n+4}+1)(\sqrt{8 n+4}-1)=$ $8 n+3$, which is coprime to $8 n+5$. Therefore, $\sqrt{8 n+4}+1$ and $\sqrt{8 n+4}-1$ are coprime to $8 n+5$.

Assume for the sake of contradiction that $\left|V_{d}\right| \geqslant 5$ for each $d \in[3]$.

Case 1: For all $d \in[3], x \in V_{d}$ implies $x+1 \notin V_{d}$.

By Lemma 20, each vertex has arcs leading to all but at most two vertices in each of the other $V_{i}$.

Suppose, without loss of generality, that there are three $x \in V_{1}$ so that $\left|\Delta_{x, 1,2}\right| \leqslant \sqrt{8 n+4}$, and let $T_{1}$ be the set of all such nodes in $V_{1}$. 
Lemma 20 implies that each $y \in V_{2}$ is the tail of some arc directed into $T_{1}$. Thus, for each $y \in V_{2},\left|\Delta_{y, 2,1}\right| \leqslant \sqrt{8 n+4}$. Let $x_{0} \in T_{1}$. Then there are at least three $z \in V_{3}$ so that $\left(x_{0}, z\right)$ is an arc - let $T_{3}$ be any set of exactly three such $z$. Lemma 20 implies that for each $y \in V_{2}$ there is a $z \in T_{3}$ so that $(y, z)$ is an arc. There are thus five arcs from $V_{2}$ to $T_{3}$, each from a different vertex in $V_{2}$. Then there is a $z_{0} \in T_{3}$ so that there are two $y \in V_{2}$ so that $\left(y, z_{0}\right)$ is an arc - let $T_{2}$ be the set of all $y \in V_{2}$ so that $\left(y, z_{0}\right)$ is an arc.

We have $\left|\Delta_{x_{0}, 1,3}\right| \geqslant \sqrt{8 n+4}$ and $\left|\Delta_{y, 2,3}\right| \geqslant \sqrt{8 n+4}$ for each $y \in V_{2}$. These imply, respectively, that $\left|\Delta_{z_{0}, 3,1}\right| \geqslant \sqrt{8 n+4}$ and $\left|\Delta_{z_{0}, 3,2}\right| \geqslant \sqrt{8 n+4}$. Thus, $\left|\Delta_{z_{0}, 3,2}\right|=\left|\Delta_{z_{0}, 3,1}\right|=\sqrt{8 n+4}$. It then follows that $\left|\Delta_{x_{0}, 1,3}\right|=\left|\Delta_{y, 2,3}\right|=$ $\sqrt{8 n+4}$ for each $y \in T_{2}$. This contradicts the claim - one of the intersections of three planes $P_{x_{0}, 1} \cap P_{y, 2} \cap P_{z_{0}, 3}$ such that $y \in T_{2}$ is not a hole.

Case 2: There is $d \in[3]$ and $x \in \mathbb{Z}_{8 n+5}$ so that $x, x+1 \in V_{d}$.

The proof in this case is based on similar ideas, but requires additional machinery developed in the original version of this paper. The interested reader is again referred to that version.

We henceforth assume, without loss of generality, that $\left|V_{1}\right| \leqslant 4, P_{0,1}$ is good and $\Delta_{0,1,2}=$ $4 n+2$.

\subsection{All good planes in the other two directions are of classes $>1$}

Here we consider an independent set $S$ in $C_{8 n+5}^{3}, n \geqslant 10$, such that $|S| \geqslant t_{n}+3$, and the assumptions made at the end of the previous subsection hold. Then inequality (11) applies and gives severe restrictions on the cardinalities of $Y$ and $Z$. The general idea is that we cannot afford a good plane of class 1 in another direction, because then the combined restrictions would become untenable. We proceed with several observations, and keep track of the cardinality restrictions that they imply.

Lemma 23. There is no $y$ so that $P_{y, 2}$ and $P_{y+1,2}$ are both full.

Proof. Assume for the sake of contradiction that there exists $y$ so that $P_{y, 2}$ and $P_{y+1,2}$ are full. Choose $z_{0}$ so that $\left(0, y, z_{0}\right)$ is a hole. Then $\left(0, y+1, z_{0}+(4 n+2)\right)$ is a hole, as is $\left((4 n+2) \Delta_{y, 2,3}, y, z_{0}+(4 n+2)\right)$. Since $P_{y, 2}$ and $P_{y+1,2}$ are both full, rows $y$ and $y+1$ are full in $P_{z_{0}+(4 n+2), 3}$. Furthermore, $(4 n+2) \Delta_{y, 2,3}$ is a divisor of $2 n+1$. Lemmas 8 and 15 imply that $|Z| \geqslant n+6 \geqslant 16$. This in turn implies, by (11), that $|Y| \geqslant 12 n-5>8 n+5$, a contradiction.

It follows from Lemma 23 and (11) that we have

$$
2 n+5 \leqslant|Y| \leqslant 4 n+2 \quad \text { and } \quad 6 \leqslant|Z| \leqslant 7 .
$$

(Note that we have proved $|S| \leqslant t_{n}+3$.)

We now consider $X$. 
Lemma 24. None of the good planes $P_{x, 1}$ of class 1 is solid.

Proof. Suppose for the sake of contradiction that $P_{0,1}$ is solid and $(0,0,0)$ is a hole (without loss of generality). Since $P_{1,1}$ and $P_{-1,1}$ each have at most two column badnesses, Lemma 18 implies that there are at least two $y \in Y^{+}$such that column $y$ is full in $P_{1,1}$ (equivalently column 1 is full in $P_{y, 2}$ ) and there are at least two $y \in Y^{-}$such that column $y$ is full in $P_{-1,1}$.

We first show that no $y \in Y^{+} \cap Y^{-}$gives a full column in both $P_{-1,1}$ and $P_{1,1}$. Suppose that there is $y \in Y^{+} \cap Y^{-}$so that both columns -1 and 1 are full in $P_{y, 2}$. Then the proof of Lemma 17 implies that there exist $z, a$ and $b$ so that $P_{y, 2}$ has holes at $(-1, z+a),(0, z)$, and $(1, z+b)$, where $a$ and $b$ are odd numbers in the interval $[-(2 n+1), 2 n+1]$. These are the only holes in these three columns, and trying to place the vertices of $S_{y, 2}$ leads to a contradiction by parity (i.e., Lemma 6 ).

Therefore, there are at least four $y \in Y^{+} \cup Y^{-}$that have the property that column 1 or column -1 is full in $P_{y, 2}$. It follows that there are at least four values of $y$ such that $\delta_{2}(y) \leqslant-n$. So, we have $|Y| \geqslant 4(n-1)+7$. This contradicts (12).

Let $s_{X}$ and $p_{X}$ be the numbers of singleton and positive streaks, respectively, in the sequence $\delta_{1}(x)$. Lemma 24 implies that all good planes of class 1 in $X$ are in streaks of length at least 2 . Since we assume $\left|V_{1}\right| \leqslant 4$, we have $s_{X} \leqslant 4$. Since $p_{X}+s_{X} \geqslant 6$ we have $p_{X} \geqslant 2$ and

$$
|X| \geqslant 8 .
$$

Now we consider the classes of the good planes in $Y$ and $Z$.

Lemma 25. We have $Y=V_{2}$ and $Z=V_{3}$.

Proof. We can immediately conclude that $Z=V_{3}$ : If $Z$ contains a plane of class 1 then 'rotating' the argument above we have $|Y| \leqslant 7$ or $|X| \leqslant 7$, which is not the case. Next, suppose $Y$ contains a plane $P_{y, 2}$ of class 1 . Then we may assume that $\Delta_{y, 2,1}=4 n+2$ (if it has the other orientation then the argument above yields $|X| \leqslant 7$, which is not the case). Since $Z=V_{3}$, we have $\left|\Delta_{z, 3,2}\right| \leqslant \frac{4 n+2}{3}$ for all $z \in Z$. So, it follows from Lemmas 17 and 18 that there are two values of $x$ such that $\delta_{1}(x) \leqslant-\frac{5 n+1}{3}$. Thus, we have

$$
s_{X}+p_{X} \geqslant 2\left(\frac{5 n+1}{3}-1\right)+6=\frac{10 n+14}{3} .
$$

On the other hand, a simple volume argument gives $p_{X} \leqslant \frac{8 n+5}{3}$, and therefore

$$
s_{X}+p_{X} \leqslant \frac{8 n+17}{3} .
$$

We have a contradiction. Therefore $Y=V_{2}$. 


\subsection{This configuration is impossible}

Based on all the information we have on the number and classes of good planes, and using Corollary 21, we will reach a contradiction. Indeed, we know by (12) and Lemma 25 that $\left|V_{2}\right| \geqslant 2 n+5 \geqslant 25$ and $\left|V_{3}\right| \geqslant 6$. Applying Corollary 21 to $V_{2}$ and $V_{3}$, and picking four elements $y_{1}, y_{2}, y_{3}, y_{4}$ of the set $U_{2}$ and an element $z$ of $U_{3}$, we have

$$
\left|\Delta_{z, 3,2}\right|=\left|\Delta_{y_{1}, 2,3}\right|=\left|\Delta_{y_{2}, 2,3}\right|=\left|\Delta_{y_{3}, 2,3}\right|=\left|\Delta_{y_{4}, 2,3}\right| .
$$

Let $\left(0, y^{\prime}, z\right)$ be the unique hole in the intersection of $P_{0,1}$ and $P_{z, 3}$. Lemma 12 implies

$$
0=\left(\Delta_{y_{i}, 2,3} \Delta_{z, 3,1} \Delta_{0,1,2}+1\right)\left(y^{\prime}-y_{i}\right)=( \pm(4 n+2)+1)\left(y^{\prime}-y_{i}\right)
$$

for $i=1,2,3,4$. But there are at most three values of $y_{i}$ that satisfy this condition, because $4 n+3$ is co-prime to $8 n+5$, and

$$
(4 n+1)\left(y^{\prime}-y_{i}\right)=0 \quad \Rightarrow \quad 3\left(y^{\prime}-y_{i}\right)=0 .
$$

This contradiction completes the proof of Theorem 2 .

\section{References}

[1] N. Alon, The Shannon capacity of a union, Combinatorica 18 (1998), 301-310.

[2] N. Alon and E. Lubetzky, Privileged users in zero-error transmission over a noisy channel, Combinatorica 27 (2007), 737-743.

[3] L. Baumert, R. McEliece, E. Rodemich, H. Rumsey, R. Stanley and H. Taylor, A Combinatorial Packing Problem, Computers in Algebra and Number Theory, Providence, American Mathematical Society; 1971, pp. 97-108.

[4] C. Berge, Graphs and Hypergraphs. Amsterdam and London, North-Holland; New York, American Elsevier, 1973.

[5] T. Bohman, A limit theorem for the Shannon capacities of odd cycles II, Proceedings of the AMS 133 (2005), 537-543.

[6] T. Bohman and R. Holzman, A nontrivial lower bound on the Shannon capacities of the complements of odd cycles, IEEE Transactions on Information Theory 49 (2003), 721-722.

[7] T. Bohman, R. Holzman and V. Natarajan, Maximum independent sets in certain powers of odd cycles, The Electronic Journal of Combinatorics 16 (2009), \#N26.

[8] M. Chudnovsky, N. Robertson, P. Seymour and R. Thomas, The strong perfect graph theorem, Annals of Math 164 (2006), 51-229.

[9] B. Codenotti, I. Gerace and G. Resta, Some remarks on the Shannon capacity of odd cycles, Ars Combin. 66 (2003), 243-257.

[10] G. Cornuéjols, The strong perfect graph theorem, Optima 70 (2003), 2-6. 
[11] W. Haemers, On some problems of Lovász concerning the Shannon capacity of a graph, IEEE Transactions on Information Theory 25 (1979), 231-232.

[12] R. S. Hales, Numerical invariants and the strong product of graphs, Journal of Combinatorial Theory - B 15 (1973), 146-155.

[13] P. Keevash and D. Mubayi, Stability theorems for cancellative hypergraphs, Journal of Combinatorial Theory - B 92 (2004), 163-175.

[14] P. Keevash and B. Sudakov, The Turán number of the Fano plane, Combinatorica 25 (2005), 561-574.

[15] J. Körner and A. Orlitsky, Zero-error information theory, IEEE Transactions on Information Theory 44 (1998), 2207-2229.

[16] L. Lovász, On the Shannon capacity of a graph, IEEE Transactions on Information Theory 25 (1979), 1-7.

[17] V. Natarajan, Independent sets in powers of odd cycles and the global min-cut problem. Ph. D. thesis. Carnegie Mellon University.

[18] M. Rosenfeld, On a problem of C. E. Shannon in graph theory, Proceedings of the AMS 18 (1967), 315-319.

[19] C. E. Shannon, The zero-error capacity of a noisy channel, IRE Transactions on Information Theory 2 (1956), 8-19.

[20] M. Simonovits, A method for solving extremal problems in graph theory, Theory of Graphs (Proc. Colloq. Tihany 1966) Academic Press, 1968, pp. 279-319. 